\title{
Approximate quantum cloning and the impossibility of superluminal information transfer
}

\author{
D. Bruß ${ }^{1}$, G.M. D'Ariano ${ }^{2}$, C. Macchiavello ${ }^{2}$, and M.F. Sacchi ${ }^{2}$ \\ ${ }^{1}$ Inst. für Theoret. Physik, Universität Hannover, Appelstr. 2, D-30167 Hannover, Germany \\ ${ }^{2}$ Theoretical Quantum Optics Group, Dipartimento di Fisica "A. Volta" \\ and INFM-Unità di Pavia, Via Bassi 6, 27100 Pavia, Italy
}

We show that non-locality of quantum mechanics cannot lead to superluminal transmission of information, even if most general local operations are allowed, as long as they are linear and trace preserving. In particular, any quantum mechanical approximate cloning transformation does not allow signalling. On the other hand, the no-signalling constraint on its own is not sufficient to prevent a transformation from surpassing the known cloning bounds. We illustrate these concepts on the basis of some examples.

\section{INTRODUCTION}

The impossibility of superluminal communication through the use of quantum entanglement has already been vividly discussed in the past, see for example [1]. Recently this topic has re-entered the stage of present research in the context of quantum cloning: the no-signalling constraint has been used to derive upper bounds for the fidelity of cloning transformations [8 11 . As the connection between approximate cloning and nosignalling is still widely debated, we aim at clarifying in this paper the quantum mechanical principles that forbid superluminal communication, and at answering the question whether they are the same principles that set limits to quantum cloning.

Our scenario throughout the paper for the attempt to transmit information with superluminal speed is the wellknown entanglement-based communication scheme [2 4]. The idea is the following: two space-like separated parties, say Alice and Bob, share an entangled state of a pair of two-dimensional quantum systems (qubits), for example the singlet state $\left|\psi_{s}\right\rangle=(|01\rangle-|10\rangle) / \sqrt{2}$. Alice encodes a bit of information by choosing between two possible orthogonal measurement bases for her qubit and performing the corresponding measurement. By the reduction postulate, the qubit at Bob's side collapses into a pure state depending on the result of the measurement performed by Alice. If a perfect cloning machine were available, Bob could now generate an infinite number of copies of his state, and therefore would be able to determine his state with perfect accuracy, thus knowing what basis Alice decided to use. In this way, transfer of information between Alice and Bob would be possible. In particular, if they are space-like separated, information could be transmitted with superluminal speed. The same transfer of information could evidently also be obtained if it were possible to determine the state of a single quan- tum system with perfect accuracy, which is also impossible 12,13 .

One might ask the question whether approximate cloning allows superluminal communication [14]: with imperfect cloning Bob can produce a number of imperfect copies, and thus get some information about his state. But this information is never enough to learn Alice's direction of measurement. This has been shown in Ref. 15 for a specific example. More generally, as we will show in this paper, the reason is that no local linear transformation can lead to transmission of information through entanglement, but any cloning operation consistent with quantum mechanics has to be linear.

The fact that non-locality of quantum entanglement cannot be used for superluminal communication, has been phrased as "peaceful coexistence" 16 between quantum mechanics and relativity, a much-cited expression. Here we emphasize that this consistency is not a coincidence, but a simple consequence of linearity and completeness of quantum mechanics. Our arguments go beyond previous work [1] 7 , as we consider the most general evolution on Alice's and Bob's side in the form of local maps.

Recently, this consistency has been exploited in order to devise new methods to derive bounds or constraints for quantum mechanical transformations \& 11]. However, in this paper we will show that the principles underlying the impossibility of 1) superluminal signalling and 2) quantum cloning beyond the optimal bound allowed by quantum mechanics [17 21], are not the same. In particular, the impossibility of information transfer by means of quantum entanglement is due only to linearity and preservation of trace of local operations.

\section{IMPOSSIBILITY OF SUPERLUMINAL COMMUNICATION}

In this section we want to show how the impossibility of superluminal communication arises by assuming only completeness and linearity of local maps on density operators.

We consider the most general scenario where Alice and Bob share a global quantum state $\rho_{A B}$ of two particles and are allowed to perform any local map, which we denote here with $\mathcal{A} \otimes \mathbb{1}$ and $\mathbb{1} \otimes \mathcal{B}$, respectively. The local map can be any local transformation, including a measurement averaged over all possible outcomes (which, in 
fact, cannot be known by the communication partner). Alice can choose among different local maps in order to encode the message " $m$ " that she wishes to transmit, namely she encodes it by performing the transformation $\mathcal{A}_{m} \otimes \mathbb{1}$ on her particle. Bob can perform a local transformation $\mathbb{1} \otimes \mathcal{B}$ on his particle (e.g. cloning) and then a local measurement $\mathbb{1} \otimes \Pi_{r}$ to decode the message $\left(\Pi_{r}\right.$ is a POVM [22,23]). The impossibility of superluminal communication in the particular case where Bob performs only a measurement has been demonstrated in Ref. [1]. Here we follow a more general approach, discussing the roles of "completeness" and linearity of any local map involved. By "completeness" of a map $\mathcal{A}$ we mean that the trace is preserved under its action, namely

$$
\operatorname{Tr}\left[\mathcal{A}\left(\rho_{A}\right)\right] \equiv \operatorname{Tr}\left[\rho_{A}\right]
$$

for any $\rho$ 24. Linearity of the map on trace-class operators of the form $|\psi\rangle\langle\phi|$, allows to extend the completeness condition to the whole Hilbert space, namely

$$
\operatorname{Tr}\left[\mathcal{A} \otimes \mathbb{1}\left(\rho_{A B}\right)\right] \equiv \operatorname{Tr}\left[\rho_{A B}\right],
$$

and analogously for the partial trace

$$
\operatorname{Tr}_{A}\left[\mathcal{A} \otimes \mathbb{1}\left(\rho_{A B}\right)\right] \equiv \operatorname{Tr}_{A}\left[\rho_{A B}\right]
$$

On Bob's side, only linearity without completeness is needed for the local map $\mathcal{B}$, leading to the equality

$$
\operatorname{Tr}_{A}\left[\mathcal{A} \otimes \mathcal{B}\left(\rho_{A B}\right)\right]=\mathcal{B} \operatorname{Tr}_{A}\left[\mathcal{A} \otimes \mathbb{1}\left(\rho_{A B}\right)\right]
$$

As we will show in the following, the above equations are the fundamental ingredients and the only requirements for local maps to prove the impossibility of superluminal communication.

We will now compute the conditional probability $p(r \mid m)$ that Bob records the result $r$ when the message $m$ was encoded by Alice:

$$
p(r \mid m)=\operatorname{Tr}\left[\mathbb{1} \otimes \Pi_{r}\left(\mathcal{A}_{m} \otimes \mathcal{B}\left(\rho_{A B}\right)\right)\right] .
$$

By exploiting Eqs. (伍) and (3) we have

$$
\begin{aligned}
p(r \mid m) & =\operatorname{Tr}_{B}\left[\Pi_{r} \mathcal{B}\left(\operatorname{Tr}_{A}\left[\mathcal{A}_{m} \otimes \mathbb{1}\left(\rho_{A B}\right)\right]\right)\right] \\
& =\operatorname{Tr}_{B}\left[\Pi_{r} \mathcal{B}\left(\operatorname{Tr}_{A}\left[\rho_{A B}\right]\right)\right] \equiv p(r) .
\end{aligned}
$$

The conditional probability is therefore independent of the local operation $\mathcal{A}_{m}$ that Alice performed on her particle, and therefore the amount of transmitted information vanishes. Note that the speed of transmission does not enter in any way, i.e. any transmission of information is forbidden 25], in particular superluminal transmission.

We want to stress that this result holds for all possible linear local operations that Alice and Bob can perform, and also for any joint state $\rho_{A B}$. In particular, it holds for any kind of linear cloning transformation performed at Bob's side (notice that ideal cloning is a non-linear map). Notice also that any operation that is physically realizable in standard quantum mechanics (completely positive map) is linear and complete, and therefore it does not allow superluminal communication.

We also emphasize here that the "peaceful coexistence" between quantum mechanics and relativity is automatically guaranteed by the linearity and completeness of any quantum mechanical process. Actually, as shown in the diagram 11, the set of local quantum mechanical maps is just a subset of the local maps that do not allow superluminal communication.

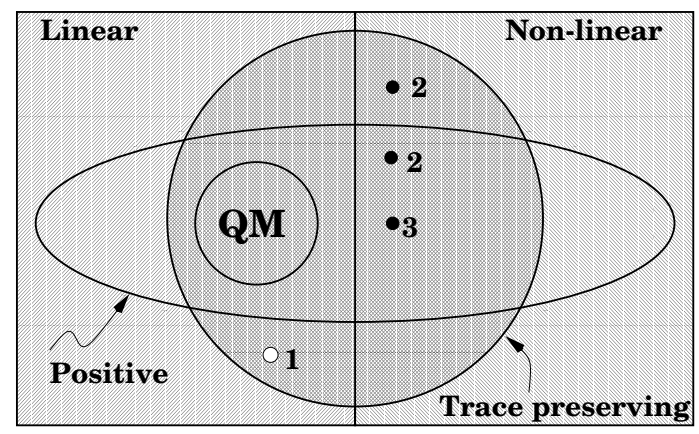

FIG. 1. Diagram of local maps. QM denotes quantum mechanical maps, namely linear trace-preserving CP maps 24. Examples from the text are placed in the diagram: map 1 (open circle) does not allow superluminal communication; map 2 and 3 (full circle) do.

In the next section we will show how superluminal communication could be achieved if one would give up the linearity requirement for the local maps, by discussing some explicit examples.

\section{EXAMPLES}

Our examples are based on the scenario where Alice and Bob share an entangled state of two qubits and Alice performs a projection measurement with her basis oriented along the direction $\vec{n}$. The final state of Bob, who does not know the result of the measurement, is given by

$$
p(\vec{n}) \rho_{\text {out }}(\vec{n})+p(-\vec{n}) \rho_{\text {out }}(-\vec{n}),
$$

where $p( \pm \vec{n})$ denote the probabilities that Alice finds her qubit oriented as $\pm \vec{n}$, and $\rho_{\text {out }}( \pm \vec{n})$ are the corresponding final density operators at Bob's side after he performed his local transformation. Notice that the evolved state of Bob, as in the following examples, can be a joint state of a composite system with more than one qubit. If the information is encoded in the choice of two possible different orientations $\vec{n}_{1}$ and $\vec{n}_{2}$ of the measurement basis, the impossibility of superluminal communication corresponds to the condition

$$
\begin{aligned}
& p\left(\vec{n}_{1}\right) \rho_{\text {out }}\left(\vec{n}_{1}\right)+p\left(-\vec{n}_{1}\right) \rho_{\text {out }}\left(-\vec{n}_{1}\right) \\
& =p\left(\vec{n}_{2}\right) \rho_{\text {out }}\left(\vec{n}_{2}\right)+p\left(-\vec{n}_{2}\right) \rho_{\text {out }}\left(-\vec{n}_{2}\right)
\end{aligned}
$$


for all choices of $\vec{n}_{1}$ and $\vec{n}_{2}$. In the following section we give some explicit examples of local maps on Bob's side. Notice that we will intentionally leave the ground of quantum mechanics (an explicit example of a superluminal communication scheme based on the use of non-linear evolutions is also given in Ref. [27]).

(1) Example of a linear, non-positive $1 \rightarrow 2$ cloning transformation which does not allow superluminal communication:

The evolved state at Bob's side after his transformation is a state of two qubits given by

$$
\begin{aligned}
& \rho_{\text {out }}(\vec{s})= \\
& \frac{1}{4}\left[\mathbb{1} \otimes \mathbb{1}+\eta(\vec{s} \cdot \vec{\sigma} \otimes \mathbb{1}+\mathbb{1} \otimes \vec{s} \cdot \vec{\sigma})+t \sum_{j=x, y, z} \sigma_{j} \otimes \sigma_{j}\right]
\end{aligned}
$$

where $\vec{s}$ is the Bloch vector which is cloned and $\eta$ is the shrinking factor. The above map is non-positive for $\eta>$ $(1+t) / 2$ [8]. This is the case, for instance, for $t=1 / 3$ and $\eta>2 / 3$. Such a transformation violates the upper bound of the $1 \rightarrow 2$ universal quantum cloner 28,18 but, as this is a linear transformation, Eq. (6) holds. Therefore the cloning is "better" than the optimal one, and the no-signalling condition (8) is still fulfilled.

This means that we can go beyond the laws of quantum mechanics (complete positivity) without necessarily creating the possibility of superluminal communication.

(2) Example of non-linear, positive or non-positive $1 \rightarrow$ 2 cloning transformation which does allow superluminal communication:

Consider Bob's transformation

$$
\begin{aligned}
\rho_{\text {out }}(\vec{s}) & =\frac{1}{4}[\mathbb{1} \otimes \mathbb{1} \\
& +\left(\sum_{j=x, y, z} f_{j}\left(s_{j}\right) \sigma_{j} \otimes \mathbb{1}+\mathbb{1} \otimes \sum_{j=x, y, z} f_{j}\left(s_{j}\right) \sigma_{j}\right) \\
& \left.+t \sum_{j=x, y, z} \sigma_{j} \otimes \sigma_{j}\right]
\end{aligned}
$$

where $f_{j}\left(s_{j}\right)$ denotes a function of the component $j$ of the Bloch vector, which is such that this map acts nonlinearly on a convex combination of density matrices. For odd functions, namely $f_{j}\left(s_{j}\right)=-f_{j}\left(-s_{j}\right)$ one does not violate the no-signalling condition for a maximally entangled state because taking $\vec{s}= \pm \vec{n}$ it follows that $\rho_{\text {out }}(\vec{n})+\rho_{\text {out }}(-\vec{n})$ does not depend on $\vec{n}$, whereas for even non-constant functions one does. However, for odd functions the no-signalling condition is in general violated for partially entangled pure states, i.e. $p(\vec{n}) \neq p(-\vec{n})$ in Eq. (7). It is interesting to see that in this non-physical case superluminal communication is achieved when sharing less than maximal entanglement.

Depending on the value of the parameter $t$ this map can be positive or non-positive. Examples of non-positive maps can for instance be found by violating the condition $f_{z}(1)>(1+t) / 2$ (compare with previous example).

(3) Example of a non-linear, positive $1 \rightarrow N$ cloning transformation which does allow superluminal communi- cation:

Consider

$$
\begin{aligned}
& |\psi\rangle\langle\psi|\otimes| 0\rangle\left\langle\left. 0\right|^{\otimes(N-1)} \rightarrow\right. \\
& F|\psi\rangle\left\langle\left.\psi\right|^{\otimes N}+(1-F) \mid \psi_{\perp}\right\rangle\left\langle\left.\psi_{\perp}\right|^{\otimes N}, \quad N \geq 2,\right.
\end{aligned}
$$

where $\left|\psi_{\perp}\right\rangle$ is orthogonal to $|\psi\rangle$. The no-signalling condition (8) for two different choices of basis $\left\{|\psi\rangle,\left|\psi_{\perp}\right\rangle\right\}$ and $\left\{|\phi\rangle,\left|\phi_{\perp}\right\rangle\right\}$ with equiprobable outcomes is violated because

$$
|\psi\rangle\left\langle\left.\psi\right|^{\otimes N}+\mid \psi_{\perp}\right\rangle\left\langle\left.\psi_{\perp}\right|^{\otimes N} \neq \mid \phi\right\rangle\left\langle\left.\phi\right|^{\otimes N}+\mid \phi_{\perp}\right\rangle\left\langle\left.\phi_{\perp}\right|^{\otimes N},\right.
$$

which holds for any value $0 \leq F \leq 1$. It is then possible to devise a measurement procedure that distinguishes between the left and right hand side of Eq. (12), thus allowing to transmit information faster than light.

In order to illustrate this we give an explicit example with $N=2$. Let us denote the right hand side of equation (11) as $\bar{\rho}(\psi)$. We choose $|\psi\rangle=|0\rangle$ and $|\phi\rangle=(|0\rangle+$ $|1\rangle) / \sqrt{2}$ and a POVM measurement on the clones given by the operators $E_{0}$ and $E_{1}$, which are the projectors over the subspaces spanned by $\{|01\rangle,|10\rangle\}$ and $\{|00\rangle,|11\rangle\}$, respectively.

With this measurement the probabilities for outcome 0 and 1 depend on Alice's choice of measurement basis. We denote as $p(0 \mid \psi)$ the probability that Bob finds outcome 0 , if Alice measured in the basis $\left\{|\psi\rangle,\left|\psi_{\perp}\right\rangle\right\}$, and arrive at

$$
\begin{aligned}
& p(0 \mid \psi)=\frac{1}{2} \operatorname{Tr}\left[E_{0}\left(|\psi\rangle\left\langle\left.\psi\right|^{\otimes 2}+\mid \psi_{\perp}\right\rangle\left\langle\psi_{\perp}\right|\right)^{\otimes 2}\right]=0 \\
& p(1 \mid \psi)=1-p(0 \mid \psi)=1
\end{aligned}
$$

Analogously, for the other choice of Alice's basis one has

$$
\begin{aligned}
& p(0 \mid \phi)=\frac{1}{2} \operatorname{Tr}\left[E_{0}\left(|\phi\rangle\left\langle\left.\phi\right|^{\otimes 2}+\mid \phi_{\perp}\right\rangle\left\langle\left.\phi_{\perp}\right|^{\otimes 2}\right)\right]=\frac{1}{2},\right. \\
& p(1 \mid \phi)=1-p(0 \mid \phi)=\frac{1}{2} .
\end{aligned}
$$

Therefore, we can distinguish between the two different choices of bases. Note that, when giving up the constraint of linearity, one could send signals superluminally even for fidelities smaller than those of optimal quantum cloning.

Similar arguments hold for the transformation

$$
\begin{aligned}
& |\psi\rangle\langle\psi|\otimes| 0\rangle\left\langle\left. 0\right|^{\otimes(N-1)} \rightarrow\right. \\
& \left(F|\psi\rangle\left\langle\psi|+(1-F)| \psi_{\perp}\right\rangle\left\langle\psi_{\perp}\right|\right)^{\otimes N} .
\end{aligned}
$$

\section{CONCLUSIONS}

We have shown that the "peaceful coexistence" between quantum mechanics and relativity is automatically 
guaranteed by the linearity and completeness (i.e. tracepreserving property) of any quantum mechanical process: hence, any approximate optimal quantum cloning, as a particular case of a linear trace-preserving map, cannot lead to signalling.

For the sake of illustration, in figure 11 we summarize the set of local maps. This set is divided into linear and non-linear maps. Any linear trace-preserving map forbids superluminal signalling. Reversely, the nosignalling condition implies only linearity, as shown in Refs. [6] and [27,26]. The positive maps contain the linear maps allowed by quantum mechanics (QM), namely the completely positive trace-preserving maps. Both trace-preservation and positivity - crucial for quantum mechanics - are not implied by the no-signalling constraint. In particular, positivity seems to be unrelated with no-signalling. Hence, there is room for maps that go beyond quantum mechanics, but still preserve the constraint of no-superluminal signalling, and Example 1) above shows that this is the case.

From what we have seen we can conclude that any bound on a cloning fidelity cannot be derived from the nosignalling constraint alone, but only in connection with other quantum mechanical principles: Example 3) shows how the cloning fidelity is unrelated to the no-signalling condition. Quantum mechanics as a complete theory, however, naturally guarantees no-signalling, and obviously gives the correct known upper bounds on quantum cloning.

\section{ACKNOWLEDGEMENTS}

We thank C. Fuchs, G. C. Ghirardi, L. Hardy and A. Peres for fruitful discussions. DB acknowledges support by the ESF Programme QIT, and from Deutsche Forschungsgemeinschaft under SFB 407 and Schwerpunkt QIV. The Theoretical Quantum Optics Group of Pavia acknowledges the European Network EQUIP and Cofinanziamento 1999 "Quantum information transmission and processing: quantum teleportation and error correction" for partial support.

[1] G. Ghirardi, A. Rimini, and T. Weber, Lett. Nuovo Cimento 27, 293 (1980).

[2] N. Herbert, Found. Phys. 12, 1171 (1982).

[3] W. K. Wootters, W. H. Zurek, Nature 299, 802 (1982).

[4] G. Ghirardi, R. Grassi, A. Rimini and T. Weber, Europhys. Lett. 6, 95 (1988).

[5] H. Scherer and P. Busch, Phys. Rev. A 47, 1647 (1993).

[6] G. Svetlichny, Found. Phys. 28, 131 (1998).

[7] A. Peres, Phys. Rev. A 61, 022117 (2000).

[8] N. Gisin, Phys. Lett. A 242, 1 (1998).
[9] L. Hardy and D.D. Song, Phys. Lett. A 259, 331 (1999).

[10] S. Ghosh, G. Car and A. Roy, Phys. Lett. A 261, 17 (1999).

[11] A.K. Pati, quant-ph/9908017.

[12] G. M. D'Ariano and H. P. Yuen, Phys. Rev. Lett. 76 2832 (1996).

[13] P. Busch, in Potentiality, Entanglement and Passion-ata-Distance: Quantum Mechanical Studies for Abner Shimony, eds. R.S. Cohen, M.A. Horne, J. Stachel, Kluwer, Dordrecht, 1997 quant-ph/9604014.

[14] This problem has been posed to us by G. C. Ghirardi.

[15] C. Simon, G. Weihs and A. Zeilinger, Acta Phys. Slovaca 49, 755 (1999).

[16] A. Shimony, in Foundations of Quantum Mechanics in the Light of New Technology, ed. S. Kamefuchi, Phys. Soc. Japan, Tokyo, 1983.

[17] N. Gisin and S. Massar, Phys. Rev. Lett. 79, 2153 (1997).

[18] D. Bruß, D. DiVincenzo, A. Ekert, C. Fuchs, C. Macchiavello and J. Smolin, Phys. Rev. A 57, 2368 (1998).

[19] R. Werner, Phys. Rev. A58, 1827 (1998).

[20] D. Bruß, A. Ekert and C. Macchiavello, Phys. Rev. Lett. 81, 2598 (1998).

[21] L. Duan, G. Guo, Phys. Rev. Lett. 80, 4999 (1998).

[22] C. W. Helstrom, Quantum Detection and Estimation Theory, Academic Press, New York, 1976.

[23] A. Peres, Quantum theory: concepts and methods, Kluwer, Dordrecht, 1993.

[24] We refer to the map $\mathcal{A}$ as complete as synonymous of trace-preserving since generally Alice's map is a measurement, and summing over all possible outcomes (i.e. for the completeness of the measurement) leads to a linear trace-preserving map (non linear state reduction maps are always linear on average).

[25] A. Peres, private communication.

[26] N. Gisin, Helv. Phys. Acta 62, 363 (1989).

[27] N. Gisin, Phys. Lett. A 143, 1 (1990).

[28] V. Bužek and M. Hillery, Phys. Rev. A 54, 1844 (1996). 